\title{
An Adaptive Neuro-Control Approach for Multi-machine Power Systems
}

\author{
Zhen Ni · Yufei Tang · Xianchao Sui · Haibo He • Jinyu Wen
}

Received: date / Accepted: date

\begin{abstract}
We investigate an adaptive neuro-control approach, namely goal representation heuristic dynamic programming (GrHDP), and study the nonlinear optimal control on the multi-machine power system. Compared with the conventional control approaches, the proposed controller conducts the adaptive learning control and assumes unknown of the power system mathematic model. Besides, the proposed design can provide an adaptive reward signal that guides the power system dynamic performance over time. In this paper, we integrate the novel neuro-controller into the multi-machine power system and provide adaptive supplementary control signals. For fair comparative studies, we include the control performance with the conventional heuristic dynamic programming (HDP) approach under the same conditions. The damping performances with and without the conventional power system stabilizer (PSS) are also presented for comparison. Simulation results verify that the investigated neuro-controller can achieve improved performance in terms of the transient stability and robustness under different fault conditions.
\end{abstract}

Keywords Multi-machine power system · transient stability · excitation control · goal representation adaptive dynamic programming.

Z. Ni, Y. Tang and H. He are with Department of Electrical, Computer and Biomedical Engineering, University of Rhode Island, Kingston, RI 02881, USA. Email:\{ni@ele.uri.edu, ytang@ele.uri.edu, he@ele.uri.edu\}.

X. Sui is with Dalian Power Supply Company, Dalian, 116033, China. Email:\{xianchaosui@gmail.com\}.

J. Wen is with the College of Electrical, Electronic and Engineering, Huazhong University of Science and Technology, Wuhan, 430074, China. Email:\{jinyu.wen@hust.edu.cn\}.

\section{Introduction}

In current electrical power and energy system, the sophisticated learning and optimization approaches have been studied and investigated among various power grid examples [1] [2] [3]. As the power system is usually a large-scale system with grid-connected power plants and various loads, the optimal control strategies for the transient stability have become more and more critical in the field. The objectives for the controller and stabilizer are to achieve satisfied reliable and efficient operation for the power grid. Power system stabilizer (PSS) was adopted in response to the power system oscillations under various faults [4] [5]. Conventional PSS was usually designed based on the linearized power system model and conducted the pole-placement technique (i.e., residue-based method) to increase damping for the system oscillations [6] [7] [8]. A comprehensive comparison of PSS, static var compensators (SVCs), and shunt static synchronous compensators (STATCOMs) was investigated from both mathematic viewpoint and simulation demonstration in [9].

Current research works and field implementations on various PSS design indicate that the PSS is a powerful and promising technique, with which the performance can be guaranteed under certain conditions. However, the PSS based supplementary control is closely related to the characteristics of the power system model. If the mathematic model function is not exactly known or even unknown, it will be very hard or even impossible to obtain the linearized system model and the subsequent PSS design. In this paper, we investigated a novel adaptive neuro-control approach for the multi-machine power system based on [10] [11]. From a viewpoint of architectural design, this investigated approach has one additional network, namely goal network, to provide 
an adaptive reward signal. In addition, the value function is also closely related to this adaptive reward signal. Compared with the conventional neuro-control approaches, this investigated approach can provide improved damping performance (e.g., shorter rising time and less overshoot) for the multi-machine power system under various conditions.

Specifically, we investigate the goal representation heuristic dynamic programming (GrHDP) approach on the multi-machine synchronization control problem. The architecture of this GrHDP is different from the traditional heuristic dynamic programming (HDP) in twofold: one is that there is an additional neural network, namely the goal network, to provide an adaptive reward signal based on the current system state. The other one is that this adaptive reward signal is also set as one of the inputs for the critic network, and thus contributes to the evaluation of the control performance. To connect the multi-machine power system, the control action generated by the GrHDP controller is set as the supplementary control signal for the excitation system in each generator. The objective of this design is to improve the damping performance of the system, such that the rotor speed of each generator can be synchronous faster after various faults or disturbances. In this paper, we demonstrate the improved damping performance over the three-machine nine-bus system with three different conditions (i.e., three-phase ground fault, step changes of output active power, and load fluctuations). For fair comparison, we provide the performance with the conventional HDP design under the same settings. The damping performances with and without PSS are also presented for reference.

The rest of the paper is organized as follows: Section 2 provides a backdrop of the related work. Section 3 provides the design and the description of the simulation platform. Section 4 presents the designed new adaptive neuro-control for the multi-machine power system. Then three case studies are provided with four approaches and simulation results are discussed in Section 5. Finally, the conclusion is presented in Section 6 .

\section{Related Work}

In recent years, researchers have been working towards the self-tuning adaptive control schemes to enhance power system dynamics and stability. For instance, the coordinated tuning of PSS was proposed and demonstrated promising performance with flexible ac transmission systems (FACTS) in [12]. The decentralized nonlinear optimal excitation control was provided with improved performance on three-machine six-bus system over both the conventional PSS and automatic voltage regulator
(AVR) [13]. In addition, the novel parameter tuning and implementation steps for PSS based excitation control were investigated in [14] [15]. Many others also proposed the optimal damping performance with their controllers through the fuzzy logic control [16] [17], particle swarm optimization (PSO) [18] [19], model predicted control [13] [20], evolutionary programming (EP) [21] and so on [22] [23] [24]. The excitation control based on various PSS designs has been demonstrated with powerful capability in the power system, including excitation control for multi-machine power systems [25] [13], the wide-area damping controller (WADC) with FACTS devices [26] [27] [28], and energy storage device (ESD) based damping control [29] [30].

Computational intelligence (CI) has been introduced into power system stability control areas, and has also shown promising performances on various applications based on adaptive dynamic programming (ADP) ${ }^{1}$ [31] [32] [33]. In ADP based controller design, the exact mathematic model function is not a prerequisite. The controller observes the input vector from the power system, and provides the supplementary control signal for the exciter. A reward signal will be provided based on the current system performance and a value function will be used to critique the performance of this control action. There are usually two neural networks in the ADP design: an action network is used to provide the control action while a critic network is used to evaluate the performance of this control action with a value function. In many cases, a model network is also adopted to identify the system dynamics. In literature, the researchers have implemented the dual heuristic dynamic programming (DHP) approach into the multi-machine turbogenerator control, and compared the performance with the conventional AVR and PSS [34]. The model network was built to represent the dynamics of the turbogenerator based on the input and output data, and the action/critic networks were trained offline to achieve shorter rise time and faster convergence to synchronous speed than that of the conventional governor and PSS. In [35], the authors investigated the coordinated reactive power control of a large wind farm and a STATCOM. The similar power system modeling and offline training for the action and critic networks were conducted. In [36], the authors proposed the model-free heuristic dynamic programming and demonstrated the promising results on general nonlinear systems. Then, in [37], the authors demonstrated this online modelfree HDP for the damping control on a four-machine two-area example, and further in China Southern Power

1 Both HDP and DHP designs mentioned are the different type of implementations in the ADP design family. Specifically, HDP design is used as a benchmark in this paper. 
Grid. This model-free adaptive control scheme was also investigated for the reactive power control on the grid connected wind-farm [38]. The further comparison between the PSS and the HDP controller on the doublyfed induction generator (DFIG) was also provided in [39]. The intelligent local area signals damping control in power system oscillations was investigated in comparison with existing intelligent controllers [40]. Many others also studied the ADP based adaptive control approach on smart grid frontier applications, including grid-connected converter [41], static compensator in multi-machine power system [42], wide area optimal control [43], and many others [44] [45] [46][47]. Among these research work, the reward signal is usually defined as the fixed (and derivable) formulas, such as the weighted linear quadric forms [34] [35] [36] [37]. We realize that the fixed or pre-defined reward function may not be a optimal choice when the system is under different operation conditions. In addition, the parameters in the reward function are significantly relying on the engineering knowledge for such system, which may not be a good thing if the system is unstructured or with uncertainties.

Therefore, it is desirable to investigate the general purpose reward function for multi-machine power system adaptive control based on ADP, specifically GrHDP technique. To this end, the past experience for engineering hand-craft reward design is not a prerequisite anymore, and the proposed one in this paper will help to overcome the uncertainties of dynamics in power system over time.

\section{GrHDP based Multi-machine Power System Simulation Platform}

The multi-machine power system modeling and the simulation platform is introduced and discussed in this section. The schematic diagram of the GrHDP controller and the three-machine nine-bus system is presented in Fig. 1. The loads $A, B$, and $C$ are provided and the admittance matrices between each line and bus are included in Table 4. The three generators G1, G2 and $G 3$ are connected through $230 k v$ transmission lines and their parameters are provided in Table 3. As one can see from the figure, the GrHDP controller observes the measurement of rotor speed from each generator, and then provide supplementary control action signals $u_{1}$, $u_{2}$, and $u_{3}$ for the multi-machine accordingly. These control signals will be applied on each generator respectively, to adjust its rotor speed. It is possible that the generator, load and transmission lines are subjected to noise and disturbance, therefore a closed-loop control system is made through this process.

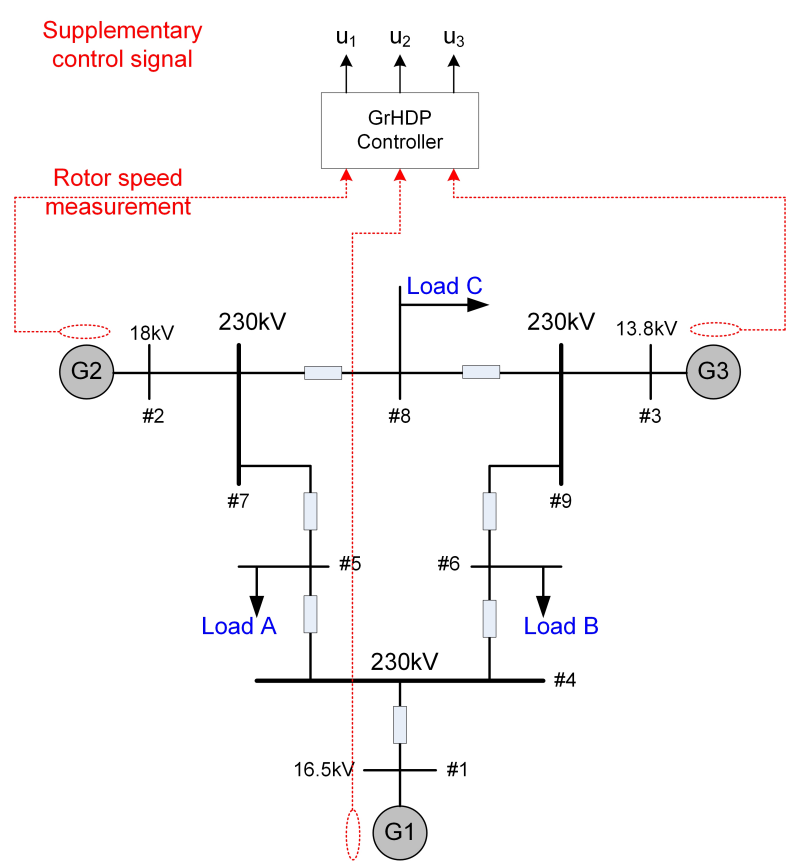

Fig. 1 The schematic diagram of the three-machine nine-bus power system. The dot lines show the observations from each generator. The GrHDP controller provides three supplementary control signals for three generators respectively.

Fig. 2 shows the control schematic diagram for a single generator. The rotor speed is transmitted from local synchronous generator and the grid (i.e., the other two synchronous generators). The rotor speed difference is then calculated and set as the input for GrHDP controller or the PSS. In the simulation studies, we also include the delayed signals of rotor speed difference for better performance. If the $S_{1}$ switches to 1 (or 2), then the GrHDP (or PSS) controller is connected in the closed-loop control system. The excitation system output $V_{\text {field }}$ will be affected by the control signal $u_{G r H D P}$ (or $u_{P S S}$ ) from the GrHDP (or PSS) controller. Eventually, the rotor speed $\omega_{1}$ is adjusted through both the governor and the excitation system.

During the regular operation, the generators are working synchronously and the parameters (e.g., the rotor speed $\omega$ and the output active power $\left.P_{e}\right)$ are at their operation set points. According to our environment settings, the inputs (i.e., the rotor speed difference) for the GrHDP controller are zero under this case. The corresponding outputs (i.e., the supplementary control signal $u_{1}, u_{2}$, and $u_{3}$ ) are also zero. This means, the GrHDP controller does not provide any supplementary control for the power system. However, during fault conditions, the GrHDP controller will provide the corresponding control signal based on the observation of rotor speed difference. Meanwhile, a proper external reward signal $r$ will be assigned as the feedback, indicating how good or 


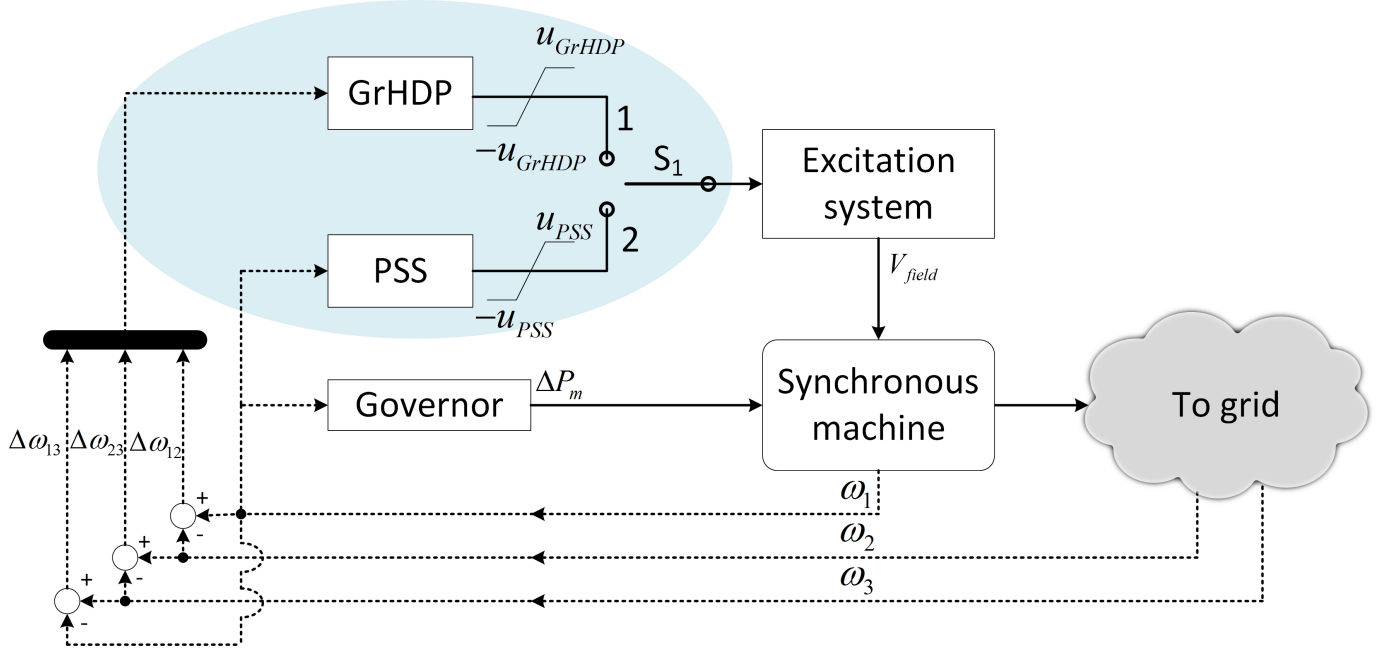

Fig. 2 The schematic diagram for a single generator. The GrHDP and PSS controller will be connected into the closed-loop if S1 switches to 1 or 2 . The dot arrows show the observed signals from the generator and the grid, while the solid arrows show the signal paths.

how bad the control performance is. Then the GrHDP controller will adjust its control action in the future. The ultimate objective for the GrHDP controller is to increase the damping and help the oscillation of $\Delta \omega$ to converge to zero as soon as possible (i.e., help the generators become synchronous operation again).

\section{Design of GrHDP Learning Controller}

\subsection{GrHDP based Control Design}

The three-network HDP learning controller was first proposed and introduced in [10] [11]. In Fig.3, the GrHDP design is presented with the conventional HDP design (lower level) and the goal representation design (upper level). The goal network provides an adaptive reward signal that can be adjusted during various operation conditions over time. As that in literature [34] [35] [37], the action network is adopted to provide supplementary control signal for the exciter, while the critic network is used to evaluate the damping performance based on the value function. In this design, instead of a fixed reward function in literature, we adopt a goal network to provide an adaptive internal reward function that can be adjusted automatically during the learning process. Moreover, the prior knowledge and the past experience of the system is not a prerequisite.

For the multi-machine power system, the action network observes the rotor speed differences between each generator, and provides the optimal supplementary control action $u$ for each exciter. The goal network observes the external reward $r$ and provides the adaptive internal reward $s$ for the critic network based on the observed input (i.e., the differences of the rotor speed). The performance of the control action will be evaluated by the critic network with a value function $J$. According to Bellman's optimality [48], the value function $J$ needs to be minimized so that the control action can achieve optimal value. In the GrHDP design, this adaptive internal reward is also set as one of the input for the critic network, and thus contributes to the value function $J$. This general reward representation helps the supplementary learning control of the multi-machine system to be more robust and fault-tolerant.

In Fig.3, the dash arrows show the objective function for each neural network and solid arrows refer to the signal paths (bolded lines refer to the vector paths). The dot dash line separates the traditional HDP design and the goal representation. The control signal $u$ provided by action network is connected with excitation system in Fig. 2. The ultimate objective for the GrHDP controller is still to solve the Bellman's optimal equation as:

$J^{*}(x, u)=\min _{u}\left(r(x, u)+\alpha J^{*}\left(x^{\prime}, u^{\prime}\right)\right)$

so that the optimal control strategy can be achieved. In (1), $x$ and $u$ refer to the current input for the action network and the control action. $x^{\prime}$ and $u^{\prime}$ refer to the one step future input for the action network and the control action. $r$ is the external reward and $\alpha$ is a discounted factor $(0<\alpha<1)$. The external reward signal $r$ is defined as:

$r(t)=-\sum_{i, j}^{N} b_{m} \Delta \omega_{i j}(t)$. 


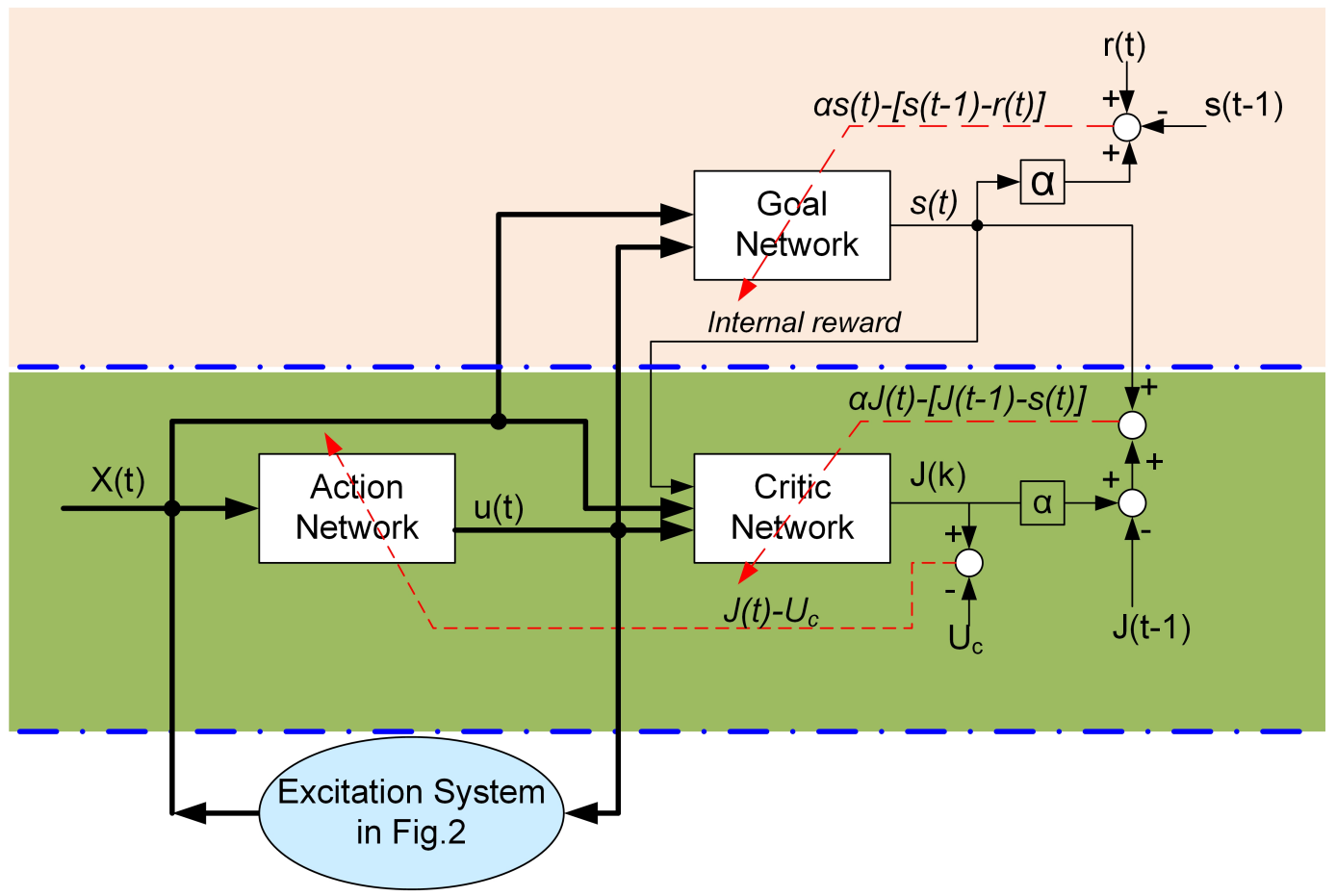

Fig. 3 The schematic diagram of the GrHDP approach. The traditional HDP approach is presented in the lower part of the figure and the goal representation is highlighted in the upper part of the figure.

where $N$ is number of generator, $\omega_{i j}(1 \leq i, j \leq N$, $i \neq j$ ) refers to the rotor speed difference between the $i-t h$ and the $j-t h$ generator. $b_{m}(1 \leq m \leq N)$ represents the weight for each component.

For the goal network, we use traditional multi-layer perceptron (MLP) neural network structure with one hidden layer. The output is used to approximate the internal goal signal as $s=f(x, u)$, and the error function can be derived from Bellman's equation by taking the temporal difference at $t-1$ and $t$ as:

$e_{g}(t)=\alpha s(t)-[s(t-1)-r(t)]$

and

$E_{g}(t)=\frac{1}{2} e_{g}^{2}(t)$

We adopt the gradient descent algorithm to adjust the weights, so that to minimize the error function of the goal network [10] [11]. The weights updating rules can be presented as:

$\frac{\partial E_{g}(t)}{\partial w_{g}(t)}=\frac{\partial E_{g}(t)}{\partial e_{g}(t)} \frac{\partial e_{g}(t)}{\partial s(t)} \frac{\partial s(t)}{\partial w_{g}(t)}$

where $w_{g}(t)$ is the weights in the goal network.

For the critic network, we use the same MLP structure as above. The output is used to approximate the total discounted utility as described in equation (1).
The error function of critic network can then be defined as:

$e_{c}(t)=\alpha J(t)-[J(t-1)-s(t)]$.

and

$E_{c}(t)=\frac{1}{2} e_{c}^{2}(t)$.

We apply the same gradient algorithm to minimize $E_{c}$ as above. Once we finish the weights-tuning in the critic network, we will start the online learning of the action network. The action network is built by the same MLP neural network structure. As the objective of the action network is to minimize the total discounted utility signal $J$, we define the error function of action network as:

$e_{a}(t)=J(t)-U_{c}$.

and

$E_{a}(t)=\frac{1}{2} e_{a}^{2}(t)$.

where $U_{c}$ is the ultimate utility function and is set as $U_{c}=0$ here. The same as the critic network, we adopt the gradient descent algorithm here to minimize (9). 


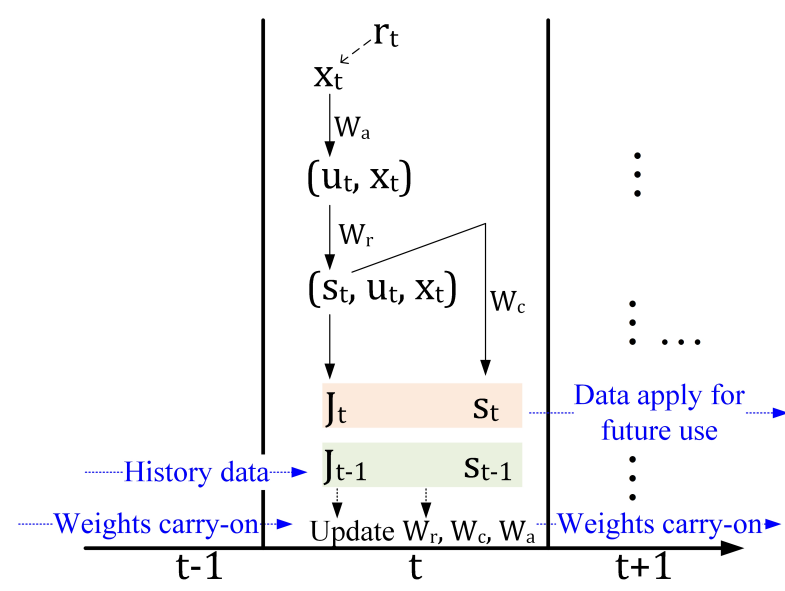

Fig. 4 The data flowchart of GrHDP controller with neural network implementation.

4.2 Flowchart of GrHDP Controller for Multi-machine Power Systems

In the multi-machine power system, the rotor differences among synchronous generators are usually chosen as the inputs for the controller. In this GrHDP based learning controller, the control outputs are used as the supplementary control signals in addition to the governors' output. The tuning parameters in the controller will be updated after the evaluation in each time step. The data flowchart has been provided in Fig. 4 and the explicit signal flowing steps are described as following:

1. At $t$ step, the rotor speed difference $x_{t}$ is observed from the multi-machine power system. The supplementary control signal $u_{t}$ can be obtained from the action network and the reward $r_{t}$ is observed.

2. The internal reinforcement signal $s_{t}$ and the value function $J_{t}$ will be calculated from the goal network and the critic network, respectively.

3. Retrieve the history data of $s_{t-1}$ and $J_{t-1}$, calculate the temporal difference errors and obtain the objective functions in both networks.

4. Update the weights parameters in the sequence of the goal network, the critic network and the action network. The weights are carried-on to the next time step.

5. Repeat from the first step when entering the $t+1$ step.

In Step 4, the update of parameters is related to the neural network weights in each network. We usually set two criteria for the learning of each network: one is the error threshold corresponding to $T_{a}, T_{c}$ and $T_{r}$ in Table 1 and the other one is the maximum iteration number corresponding to $N_{a}, N_{c}$ and $N_{r}$ in Table 1. For instance, if the squared error is minimized un- der the threshold or the iteration number exceeds the tolerance, we terminate the learning in this network. Once the weights updating of the goal, the critic and the action networks have been finished, we complete the learning process of the GrHDP controller. Generally, the selection of these parameters are important for the learning control performance, and they are explained with specific values in the simulation studies below.

\section{Simulation Studies}

For fair comparison, we compare the GrHDP controller with the conventional HDP controller under the same settings for the three-machine nine-bus system [4] [5]. In addition, we also include the performance with and without the conventional PSS controller under the same faults. As mentioned in literature [34] [37], HDP learning controllers need to learn offline for the optimized parameters. We follow the trend and let the GrHDP and HDP controller learn offline for the first trial and then test their final control policy online in the second trial. We conduct three faults in the simulation studies: the first one is the three-phase-ground fault at line 4 , the second one is the $\pm 5 \%$ step change for the output active power in generator 1 , and the third one is the sequential load fluctuations. The learning ability is compared in the first case and the robustness is checked in the second and the third cases. The admittance matrices between each line and bus for the system and the per unit values for each generator are provided in Table 4 and Table 3, respectively, in the Appendix. The parameters for the PSS is $K_{s}=0.015, T_{w}=1.5, T_{1}=0.3$ and $T_{2}=0.06$, and the output limiter is $\pm 0.035 \mathrm{pu}$ [28].

\subsection{Case 1: Three-phase-ground Fault}

A single three-phase-ground fault is applied on the line 4 at $0.5 s$, and last for $0.1 s$ with tripping the line. At $1.1 s$, the line is re-closed. We define that:

$-\Delta \omega_{12}=\omega_{1}-\omega_{2}$ : the difference of the rotor speed between the generator 1 (G1) and the generator 2 (G2);

$-\Delta \omega_{13}=\omega_{1}-\omega_{3}$ : the difference of the rotor speed between the generator $1(\mathrm{G} 1)$ and the generator 3 (G3);

$-\Delta \omega_{23}=\omega_{2}-\omega_{3}$ : the difference of the rotor speed between the generator $2(\mathrm{G} 2)$ and the generator 3 (G3); 
The input for the HDP controller is defined as:

$$
\begin{aligned}
& x(t)=\left[\begin{array}{lll}
\Delta \omega_{12}(t) & \Delta \omega_{13}(t) & \Delta \omega_{23}(t)
\end{array}\right. \\
& \Delta \omega_{12}(t-1) \Delta \omega_{13}(t-1) \quad \Delta \omega_{23}(t-1) \\
& \left.\Delta \omega_{12}(t-2) \quad \Delta \omega_{13}(t-2) \quad \Delta \omega_{23}(t-2)\right] .
\end{aligned}
$$

and the output of the HDP controller is defined as:

$u_{H D P}(t)=\left[\begin{array}{lll}u_{1}(t) & u_{2}(t) & u_{3}(t)\end{array}\right]$.

where the control action $u_{i}(t)(1 \leq i \leq 3)$ is applied to each generator respectively, and the weighted factors in (2) are set as:

$b_{1}=b_{2}=b_{3}=0.4$;

$b_{4}=b_{5}=b_{6}=0.3$;

$b_{7}=b_{8}=b_{9}=0.3$.

In literature, the coefficients for the most recent squared errors (i.e., $b_{1}, b_{2}$ and $b_{3}$ ) will be defined with a relatively large value and the coefficients for the previous squared errors (i.e., $b_{4}, b_{5}, b_{6}, b_{7}, b_{8}$ and $b_{9}$ ) will be defined with a relatively small value. These coefficients can usually sum up to 1 (i.e., $b_{1}+b_{4}+b_{7}=1$ ) as suggested in [37] [40]. The performance of both the GrHDP and the HDP controllers are based on their trained weights in neural networks (i.e., trail 2). The parameters for the GrHDP controller are provided in Table $1 . l_{*}$ indicates learning rate of the neural network. $N_{*}$ is used as maximum iteration number of the back-propagation, and $T_{*}$ is used as the error threshold of the objective function, which have been discussed in section 4.2. As there is no goal network in HDP controller, and thus it only shares the parameter values in the action network and the critic network.

Typical learning process of the GrHDP controller includes two trials described as that in [37] [49] [39]. In trial 1, the neural networks were initialized with random weights and the supplementary control outputs might not be in accordance with the desired actions. The simulation was terminated when the system reached the failure criterion. In this process, the GrHDP controller hopefully leaned a considerable amount of useful information about the state-action pairs. In trial 2, the fully trained control policies could be achieved after the first trial process. Additional online learning in trail 2 can obtain better control performance over time.

We provide the damping performance of $\Delta \omega_{12}, \Delta \omega_{13}$ and $\Delta \omega_{23}$ in Fig. 5, Fig. 6 and Fig. 7, respectively. Among the four approaches in the simulation, we can see that the GrHDP and HDP controller can achieve faster convergence to synchronous speed than that with the other two conventional control strategies. Between the GrHDP and HDP control approaches, the GrHDP
Table 1 Summary of the parameters used in the GrHDP and HDP controller.

\begin{tabular}{lcccccc}
\hline $\begin{array}{cccc}\text { Para. } \\
\text { value }\end{array}$ & $l_{c}$ & $l_{a}$ & $l_{g}$ & $N_{c}$ & $N_{a}$ \\
& 0.01 & 0.01 & 50 & 50 \\
\hline Para. & $N_{g}$ & $T_{c}$ & $T_{a}$ & $T_{g}$ & $\alpha$ \\
value & 20 & $1 e-7$ & $1 e-6$ & $1 e-7$ & 0.95 \\
$l_{*}:$ & Learning rate of the neural network; \\
$N_{*}:$ Max iteration number of the back-propagation; \\
$T_{*}:$ Error threshold of the objective function; \\
$\alpha:$ Discount factor.
\end{tabular}

controller shows less overshoot and faster convergence to synchronous speed. The robustness and online adaptation capacities of both GrHDP and HDP control approaches will be further investigated in the following two study cases.

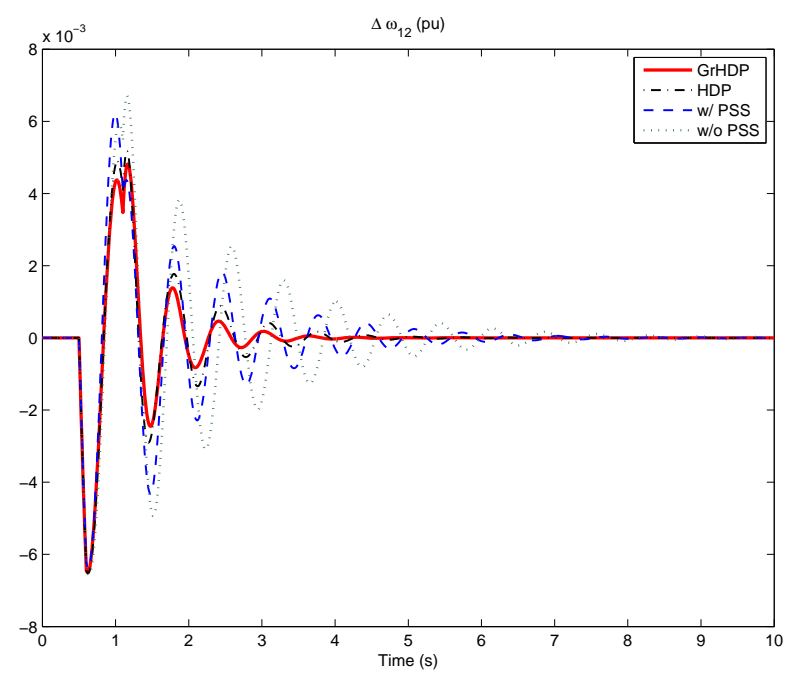

Fig. 5 Damping performance on $\Delta \omega_{12}$ under three-phaseground fault. Four approaches are compared on the same environment settings.

5.2 Case 2: $\pm 5 \%$ Step Changes for the Output Active Power

In this case study, we keep the GrHDP and HDP approaches the same learned parameters as those in section 5.1 and check the robustness for both GrHDP and HDP approaches. While for the conventional PSS, we also keep the same parameters as those in section 5.1 for comparison. The $-5 \%$ step change of the output active power is added for the generator 1 at $0.5 \mathrm{~s}$ and the $+5 \%$ step change of the output active power is added for the generator 1 at $8 \mathrm{~s}$. We present the performance of output active power $P_{e 1}, P_{e 2}$ and $P_{e 3}$ in Fig. 8, Fig. 9 and Fig. 10, respectively. An interesting observation from these three figures is that the conventional PSS 


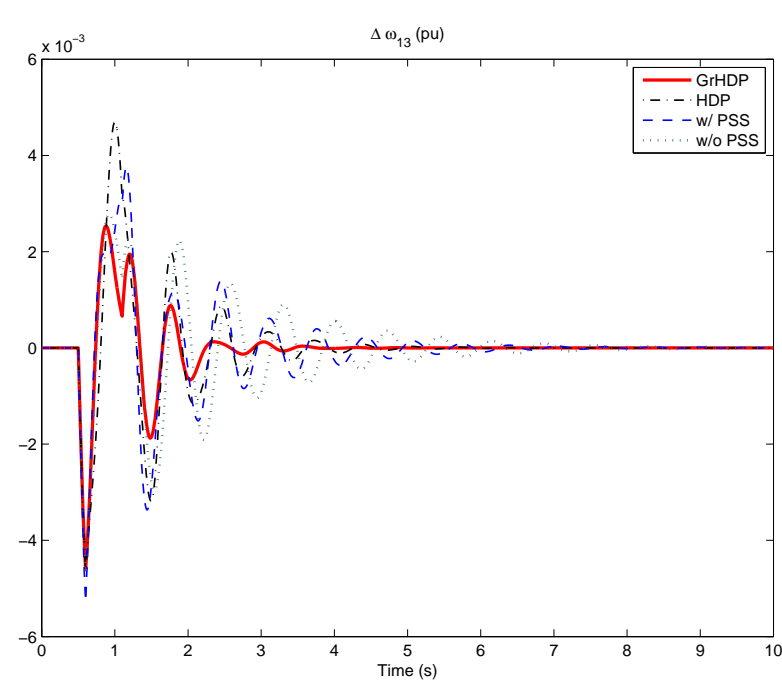

Fig. 6 Damping performance on $\Delta \omega_{13}$ under three-phaseground fault. Four approaches are compared on the same environment settings.

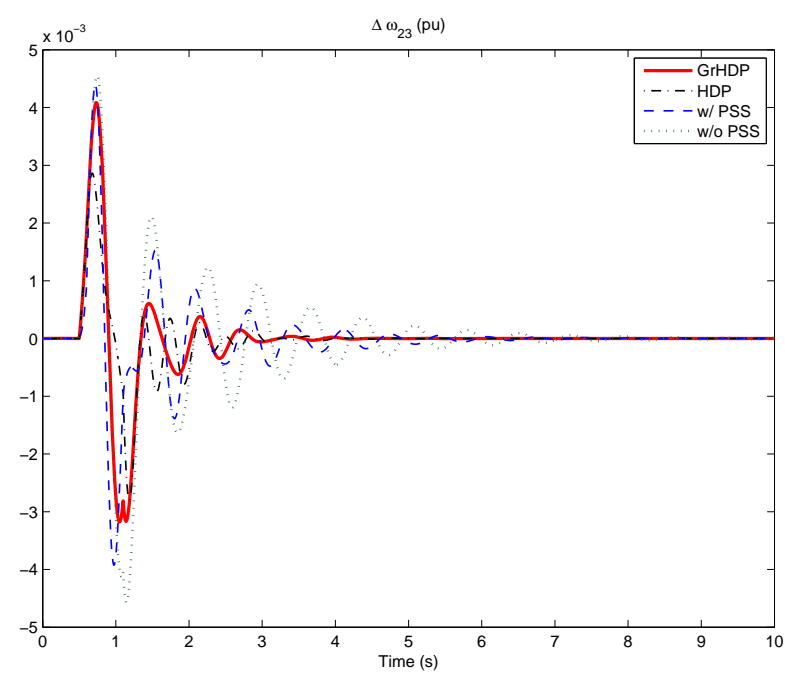

Fig. 7 Damping performance on $\Delta \omega_{23}$ under three-phaseground fault. Four approaches are compared on the same environment settings.

controller does not help the generator to go back to the original operation point. The reason is that the PSS is usually dedicated for specific fault. If the fault changes, then the PSS controller may not be able to achieve expected performance. For instance, the PSS designed for three-phase ground fault is not a good choice for the step change of output active power. While for the HDP and GrHDP controllers, both of them can achieve promising control performance. The GrHDP controller achieves shorter rising time for the output active power and less overshoot after the step changes. In Fig.10, it seems that the step changes on generator 1 do not have a significant impact on generator 3, while the designed GrHDP and HDP controllers can still show bet- ter performance compared with the conventional PSS controllers.

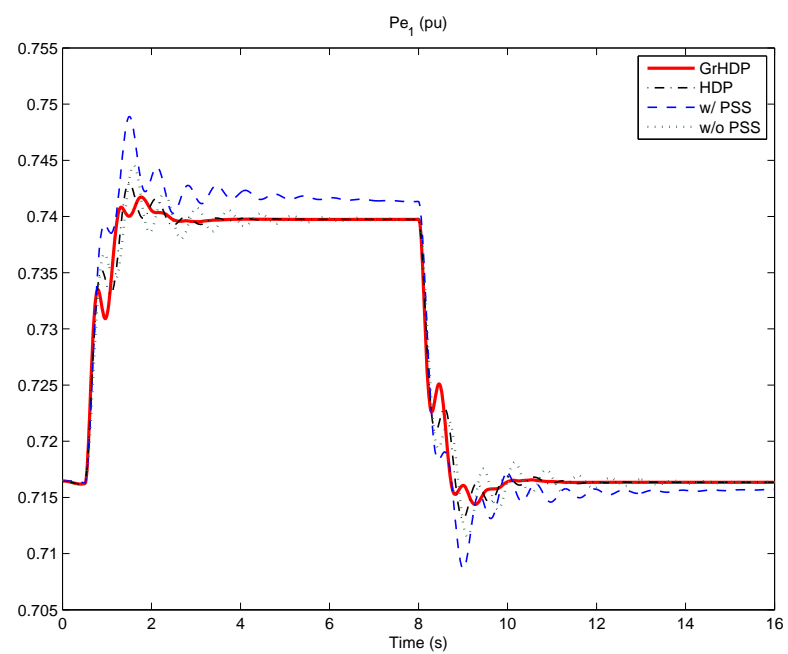

Fig. 8 Comparison of the control performance on the output active power $P e_{1}$ under both $-5 \%$ and $+5 \%$ step changes

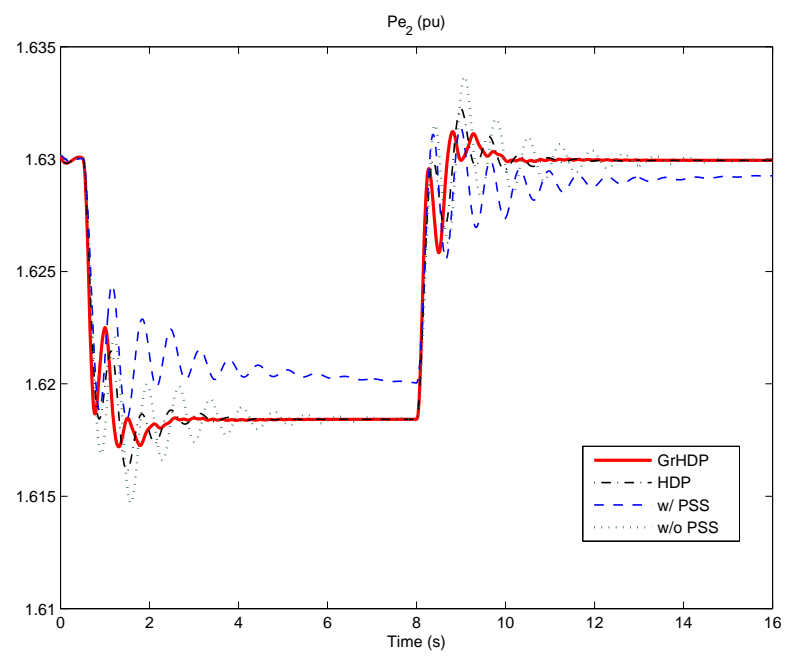

Fig. 9 Comparison of the control performance on the output active power $\mathrm{Pe}_{2}$ under both $-5 \%$ and $+5 \%$ step changes.

\subsection{Case 3: Sequential Load Fluctuations}

We further test our investigated GrHDP control approach for a sequential load fluctuation changes together with the other three approaches. The GrHDP and HDP approaches are tested based on the same weight parameters as those in section 5.2, and the PSS is also applied based on the same designed parameters in section 5.2. The load fluctuation is added for load 


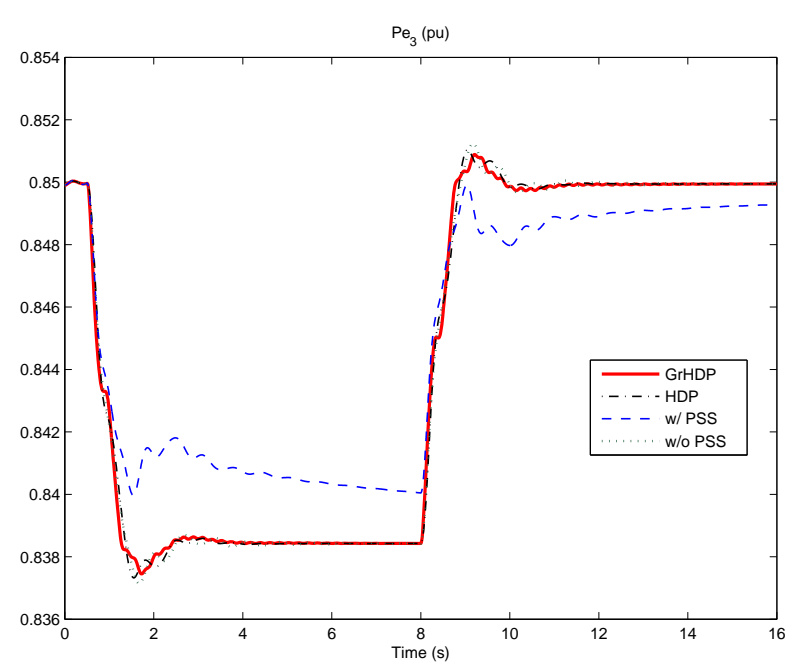

Fig. 10 Comparison of the control performance on the output active power $\mathrm{Pe}_{3}$ under both $-5 \%$ and $+5 \%$ step changes.

Table 2 The sequence of load fluctuation applied in load $A$

\begin{tabular}{lccccc}
\hline Time & $0.5 s$ & $6 s$ & $12 s$ & $18 s$ & $24 s$ \\
\hline Disturbance & $8.4 \%$ & $2.8 \%$ & $-7.0 \%$ & $-2.8 \%$ & $-1.4 \%$ \\
\hline
\end{tabular}

$A$ in a sequence of random disturbances as described in Table 2. The disturbances are presented as the percentage of the load and the positive or negative sign indicate adding or subtracting on the load. The simulation length is set as $30 \mathrm{~s}$ and assume that the output active power has been stabilized before the next disturbance happens.

The simulation results of the output active power $P e_{1}$ are provided in Fig.11. It is interesting to observe that the GrHDP can achieve very consistent control performance as those in section 5.2. In this case, the GrHDP approach can still demonstrate better performance in terms of overshoot and rise-time than any other approach. In Fig. 12, the supplementary control signals provided by GrHDP approach are also presented under this sequential load disturbances. As discussed in section 3 , the convergence of $u_{1}, u_{2}$ and $u_{3}$ further indicates that the investigated approach is stable and effective.

\section{Conclusion}

In this paper, we integrate the GrHDP learning controller into the multi-machine power system. We discuss its learning mechanism and signal flowchart, followed by the supplementary control platform for the three-machine nine-bus power system. Three case studies are conducted from a specific fault to a sequence of random disturbances. The investigated GrHDP demon-

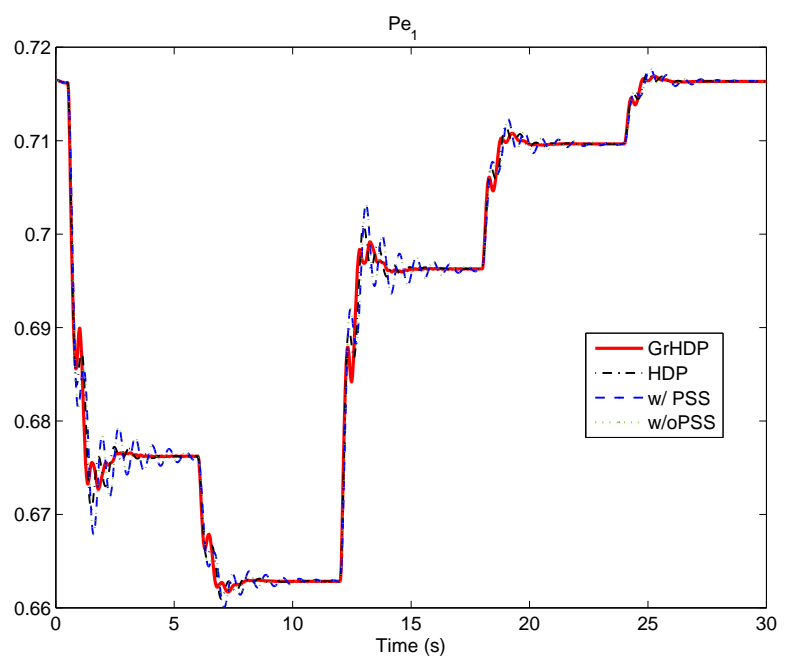

Fig. 11 The output active power $P e_{1}$ under the load fluctuations. The GrHDP approach is compared against the other three approaches under the same sequential load disturbances.
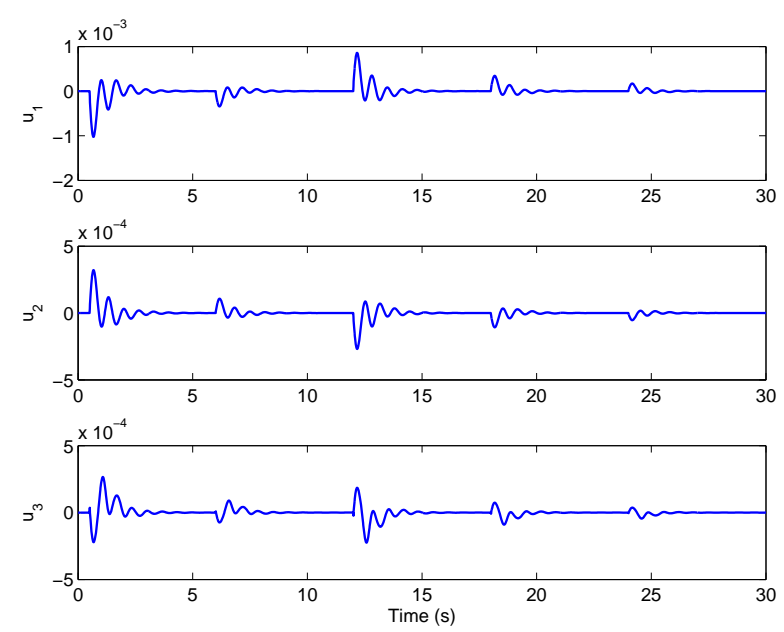

Fig. 12 The supplementary control signals $u_{1}, u_{2}$ and $u_{3}$ provided by GrHDP approach in this sequential load disturbances scenario.

strates promising learning control performance in terms of robustness and adaptation in all case studies here.

\section{Acknowledgment}

The authors gratefully acknowledge the support from the National Science Foundation under grant CAREER ECCS 1053717, the Army Research Office under grant W911NF-12-1-0378, the NSF-DFG Collaborative Research on "Autonomous Learning", a supplement grant to CNS 1117314, and the National Natural Science Foundation of China under grant 51228701 . 


\section{Appendix}

Table 3 The per unit value for the parameters of each generator/bus in the system.

\begin{tabular}{cccc}
\hline & $\mathrm{G} 1 / \# 1$ & $\mathrm{G} 2 / \# 2$ & $\mathrm{G} 3 / \# 3$ \\
$X_{d}$ & 0.146 & 0.8958 & 1.3125 \\
$X_{d}^{\prime}$ & 0.0608 & 0.1198 & 0.1813 \\
$X_{q}$ & 0.0969 & 0.8654 & 1.2578 \\
$X_{q}^{\prime}$ & 0.0969 & 0.1969 & 0.25 \\
$X_{l}$ & $8.96 s$ & $6 s$ & $5.89 s$ \\
$T_{d 0}^{\prime}$ & 0.0969 & 0.8654 & 1.2578 \\
$T_{q 0}^{\prime}$ & $0 s$ & $0.535 s$ & $0.6 s$ \\
$H$ & $23.64 s$ & $6.4 s$ & $3.01 s$ \\
$D$ & 0 & 0 & 0 \\
\hline
\end{tabular}

Table 4 The admittance matrices between each line/bus in the three-machine nine-bus system.

\begin{tabular}{ll}
\hline$Z(1,1)=0-17.3611 i$ & $Z(4,1)=0+17.3611 i$ \\
$Z(2,2)=0-16.0000 i$ & $Z(7,2)=0+16.0000 i$ \\
$Z(3,3)=0-17.0648 i$ & $Z(9,3)=0+17.0648 i$ \\
$Z(1,4)=0+17.3611 i$ & $Z(4,4)=3.3074-39.3089 i$ \\
$Z(5,4)=-1.3652+11.6041 i$ & $Z(6,4)=-1.9422+10.5107 i$ \\
$Z(4,5)=-1.3652+11.6041 i$ & $Z(5,5)=3.8138-17.8426 i$ \\
$Z(7,5)=-1.1876+5.9751 i$ & $Z(4,6)=-1.9422+10.5107 i$ \\
$Z(6,6)=4.1018-16.1335 i$ & $Z(9,6)=-1.2820+5.5882 i$ \\
$Z(2,7)=0+16.0000 i$ & $Z(5,7)=-1.1876+5.9751 i$ \\
$Z(7,7)=2.8047-35.4456 i$ & $Z(8,7)=-1.6171+13.6980 i$ \\
$Z(7,8)=-1.6171+13.6980 i$ & $Z(8,8)=3.7412-23.6424 i$ \\
$Z(9,8)=-1.1551+9.7843 i$ & $Z(3,9)=0+17.0648 i$ \\
$Z(6,9)=-1.2820+5.5882 i$ & $Z(8,9)=-1.1551+9.7843 i$ \\
$Z(9,9)=2.4371-32.1539 i$ &
\end{tabular}

$Z(9,9)=2.4371-32.1539 i$
8. P. W. Sauer and M. Pai, Power system dynamics and stability. Prentice Hall, NJ, 1998.

9. N. Mithulananthan, C. A. Canizares, J. Reeve, and G. J. Rogers, "Comparison of PSS, SVC, and STATCOM controllers for damping power system oscillations," Power Systems, IEEE Transactions on, vol. 18, no. 2, pp. 786792, 2003.

10. H. He, Z. Ni, and J. Fu, "A three-network architecture for on-line learning and optimization based on adaptive dynamic programming," Neurocomputing, vol. 78 , no. 1 , pp. 3-13, 2012.

11. H. He, Self-Adaptive Systems for Machine Intelligence. Wiley, 2011.

12. M. Furini, A. Pereira, and P. Araujo, "Pole placement by coordinated tuning of power system stabilizers and facts-pod stabilizers," International Journal of Electrical Power \&6 Energy Systems, vol. 33, no. 3, pp. 615-622, 2011.

13. W. Yao, L. Jiang, J. Fang, J. Wen, and S. Cheng, "Decentralized nonlinear optimal predictive excitation control for multi-machine power systems," International Journal of Electrical Power ES Energy Systems, vol. 55, pp. 620 627, 2014.

14. H. Bevrani, T. Hiyama, and H. Bevrani, "Robust PID based power system stabiliser: design and real-time implementation," International Journal of Electrical Power E Energy Systems, vol. 33, no. 2, pp. 179-188, 2011.

15. A. Dysko, W. E. Leithead, and J. O'Reilly, "Enhanced power system stability by coordinated pss design," Power Systems, IEEE Transactions on, vol. 25, no. 1, pp. 413422, 2010.

16. J. Wen, S. Cheng, and O. Malik, "A synchronous generator fuzzy excitation controller optimally designed with a genetic algorithm," in Power Industry Computer Applications., 1997. 20th International Conference on, pp. 106-111, IEEE, 1997.

17. K. Saoudi and M. Harmas, "Enhanced design of an indirect adaptive fuzzy sliding mode power system stabilizer for multi-machine power systems," International Journal of Electrical Power \&6 Energy Systems, vol. 54, pp. 425431, 2014.

18. H. E. Mostafa, M. A. El-Sharkawy, A. A. Emary, and K. Yassin, "Design and allocation of power system stabilizers using the particle swarm optimization technique for an interconnected power system," International Journal of Electrical Power \& Energy Systems, vol. 34, no. 1, pp. 57-65, 2012.

19. Y. Tang, P. Ju, H. He, C. Qin, and F. Wu, "Optimized control of DFIG-based wind generation using sensitivity analysis and particle swarm optimization," IEEE Trans. Smart Grid, vol. 4, no. 1, pp. 509-520, 2013.

20. B. Wu and O. P. Malik, "Multivariable adaptive control of synchronous machines in a multimachine power system," Power Systems, IEEE Transactions on, vol. 21, no. 4, pp. 1772-1781, 2006.

21. S.-K. Wang, J.-P. Chiou, and C.-W. Liu, "Parameters tuning of power system stabilizers using improved ant direction hybrid differential evolution," International Journal of Electrical Power \& Energy Systems, vol. 31, no. 1, pp. 34-42, 2009.

22. C.-X. Dou, J. Yang, X. Li, T. Gui, and Y. Bi, "Decentralized coordinated control for large power system based on transient stability assessment," International Journal of Electrical Power EJ Energy Systems, vol. 46, pp. 153-162, 2013.

23. J. Talaq, "Optimal power system stabilizers for multi machine systems," International Journal of Electrical bilizers part ii: Performance objectives and tuning contions on, no. 6, pp. 3025-3033, 1981. 
Power \& Energy Systems, vol. 43, no. 1, pp. 793-803, 2012.

24. M. Mahmud, H. Pota, and M. Hossain, "Full-order nonlinear observer-based excitation controller design for interconnected power systems via exact linearization approach," International Journal of Electrical Power \&6 Energy Systems, vol. 41, no. 1, pp. 54-62, 2012.

25. P. Zhao, W. Yao, J. Wen, L. Jiang, S. Wang, and S. Cheng, "Improved synergetic excitation control for transient stability enhancement and voltage regulation of power systems," International Journal of Electrical Power \& Energy Systems, vol. 68, pp. 44 - 51, 2015.

26. W. Yao, L. Jiang, Q. Wu, J. Wen, and S. Cheng, "Delaydependent stability analysis of the power system with a wide-area damping controller embedded," Power Systems, IEEE Transactions on, vol. 26, no. 1, pp. 233-240, 2011.

27. W. Yao, L. Jiang, J. Wen, Q. Wu, and S. Cheng, "Widearea damping controller of FACTS devices for interarea oscillations considering communication time delays," Power Systems, IEEE Transactions on, vol. 29, no. 1, pp. 318-329, 2014.

28. W. Yao, J. Wen, S. Chen, and L. Jiang, "Development of a matlab/simulink based power system simulation toolbox," Power System Technology, vol. 36, no. 6, pp. 95101, 2012.

29. J. Fang, W. Yao, Z. Chen, J. Wen, and S. Cheng, "Design of anti-windup compensator for energy storage-based damping controller to enhance power system stability," Power Systems, IEEE Transactions on, 2014, in press.

30. X. Sui, Y. Tang, H. He, and J. Wen, "Energy-storagebased low-frequency oscillation damping control using particle swarm optimization and heuristic dynamic programming," Power Systems, IEEE Transactions on, vol. 29, pp. 2539-2548, Sept 2014.

31. P. Werbos, "Computational intelligence for the smart grid-history, challenges, and opportunities," Computational Intelligence Magazine, IEEE, vol. 6, no. 3, pp. 1421, 2011.

32. R. G. Harley and J. Liang, "Computational intelligence in smart grids," in Computational Intelligence Applications In Smart Grid (CIASG), 2011 IEEE Symposium on, pp. 59-65, IEEE, 2011.

33. G. K. Venayagamoorthy, K. Rohrig, and I. Erlich, "One step ahead: short-term wind power forecasting and intelligent predictive control based on data analytics," Power and Energy Magazine, IEEE, vol. 10, no. 5, pp. 70-78, 2012.

34. G. K. Venayagamoorthy, R. G. Harley, and D. C. Wunsch, "Comparison of heuristic dynamic programming and dual heuristic programming adaptive critics for neurocontrol of a turbogenerator," IEEE Transactions on Neural Networks, vol. 13, no. 3, pp. 764-773, 2002.

35. W. Qiao, R. G. Harley, and G. K. Venayagamoorthy, "Coordinated reactive power control of a large wind farm and a statcom using heuristic dynamic programming," Energy Conversion, IEEE Transactions on, vol. 24, no. 2, pp. 493-503, 2009.

36. J. Si and Y.-T. Wang, "Online learning control by association and reinforcement," Neural Networks, IEEE Transactions on, vol. 12, no. 2, pp. 264-276, 2001.

37. C. Lu, J. Si, and X. Xie, "Direct heuristic dynamic programming for damping oscillations in a large power system," Systems, Man, and Cybernetics, Part B: Cybernetics, IEEE Transactions on, vol. 38, no. 4, pp. 1008-1013, 2008.
38. Y. Tang, H. He, Z. Ni, J. Wen, and X. Sui, "Reactive power control of grid-connected wind farm based on adaptive dynamic programming," Neurocomputing, vol. 125, pp. 125-133, Feb. 2014.

39. Y. Tang, H. He, and J. Wen, "Comparative study between hdp and pss on dfig damping control," in Computational Intelligence Applications In Smart Grid (CIASG), 2013 IEEE Symposium on, pp. 59-65, IEEE, 2013.

40. D. Molina, G. K. Venayagamoorthy, J. Liang, and R. G. Harley, "Intelligent local area signals based damping of power system oscillations using virtual generators and approximate dynamic programming," Smart Grid, IEEE Transactions on, vol. 4, no. 1, pp. 498-508, 2013.

41. X. Fu, S. Li, M. Fairbank, D. C. Wunsch, and E. Alonso, "Training recurrent neural networks with the levenbergmarquardt algorithm for optimal control of a gridconnected converter," Neural Networks and Learning Systems, IEEE Transactions on, 2015, in press.

42. S. Mohagheghi, Y. Del Valle, G. K. Venayagamoorthy, and R. G. Harley, "A proportional-integrator type adaptive critic design-based neurocontroller for a static compensator in a multimachine power system," Industrial Electronics, IEEE Transactions on, vol. 54, no. 1, pp. 8696, 2007.

43. S. Mohagheghi, G. K. Venayagamoorthy, and R. G. Harley, "Optimal wide area controller and state predictor for a power system," Power Systems, IEEE Transactions on, vol. 22, no. 2, pp. 693-705, 2007.

44. C. Wei, Z. Zhang, W. Qiao, and L. Qu, "Reinforcement learning-based intelligent maximum power point tracking control for wind energy conversion systems," Industrial Electronics, IEEE Transactions on, 2015, in press.

45. Y. Tang, H. He, Z. Ni, and J. Wen, "Optimal operation for energy storage with wind power generation using adaptive dynamic programmin," in Proc. IEEE Power and Energy Society General Meeting (IEEE PES General Meeting'15), pp. 1-6, Jul., Denver, CO.

46. Z. Ni, Y. Tang, H. He, and J. Wen, "Multi-machine power system control based on dual heuristic dynamic programming," in Proc. of 2014 IEEE Symposium on Computational Intelligence Applications in Smart Grid (CIASG), pp. 1-7, Dec. 2014, Orlando, FL.

47. Y. Tang, J. Yang, J. Yan, and H. He, "Intelligent load frequency controller using GrADP for island smart grid with electric vehicles and renewable resources," Neurocomputing, 2015.

48. R. Bellman, Dynamic Programming. Princeton, NJ: Princeton Univ. Press, 1957.

49. Y. Tang, H. He, J. Wen, and J. Liu, "Power system stability control for a wind farm based on adaptive dynamic programming," Smart Grid, IEEE Transactions on, vol. 6 , no. 1 , pp. $166-177,2015$. 